\title{
Ketogenic Diets: Evidence for Short- and Long-term Efficacy
}

\author{
Eric H. Kossoff* and Jong M. Rho ${ }^{\dagger}$ \\ *John M. Freeman Pediatric Epilepsy Center, Departments of Neurology and Pediatrics, Johns Hopkins Hospital, Baltimore, \\ Maryland 21287, and ${ }^{\dagger}$ The Barrow Neurological Institute and St. Joseph's Hospital and Medical Center, Phoenix, \\ Arizona 85013
}

\begin{abstract}
Summary: The use of dietary treatments for epilepsy (ketogenic, modified Atkins, and low glycemic index diets) has been in continuous use since 1921. These treatments have been well studied in the short term, with approximately half of children having at least a 50\% reduction in seizures after 6 months. Approximately one third will attain $>90 \%$ reduction in their seizures. Animal studies confirm these findings, with broad evidence demonstrating acute anticonvulsant effects of the diet. Furthermore, the diet appears to maintain its efficacy in humans
\end{abstract}

when provided continuously for several years. Interestingly, benefits may be seen long term even when the diet is discontinued after only a few months of use, suggesting neuroprotective effects. This potential antiepileptogenic activity has been recently demonstrated in some animal studies as well. This review discusses the animal and human evidence for both short- and long-term benefits of dietary therapies. Key Words: Ketogenic diet, clinical efficacy, short-term, long-term, ketone bodies, fatty acids.

\section{INTRODUCTION}

Compared with most other nonpharmacologic therapies, the use of dietary therapy for epilepsy is simple, technologically. It has been in continuous use for nearly 90 years, supported by hundreds of publications describing potential benefits. ${ }^{1}$ Although used primarily for children, diets may have a significant role in the future for adults and benefits may be identified for neurologic conditions other than epilepsy.

What are the primary dietary therapies for epilepsy? At this time, these therapies include the traditional ketogenic diet (KD), the modified Atkins diet, and the low glycemic index treatment. All three diets have been studied and are in use for both children and adults worldwide.

The KD, first introduced in $1921,{ }^{2}$ is high in fat, adequate for protein $(1 \mathrm{~g} / \mathrm{kg}$ per day), and low in carbohydrate; the diet induces high levels of serum $\beta$-hydroxybutyrate (and urinary acetoacetate). It is calorie and fluid restricted and traditionally is started in the hospital after a 1- to 2-day fasting period. ${ }^{3}$ The KD can be provided as the classic diet, with predominantly long-chain triglycerides, or in a modification using gradually increasing percentages of medium-chain triglycerides.

Address correspondence and reprint requests to: Dr. Eric H. Kossoff, The Johns Hopkins Hospital, Suite 2158, 200 North Wolfe Street, Baltimore, MD 21287. E-mail: ekossoff@jhmi.edu.
The modified Atkins diet was introduced in 2003 and mimics the KD in composition but without calorie, protein, or fluid restriction. ${ }^{4}$ Carbohydrates are restricted to $10 \mathrm{~g}$ to $15 \mathrm{~g}$ per day, and this diet is started on an outpatient basis without a fasting period. Similarly, the low glycemic index treatment is an outpatient-initiated therapy without restrictions on calories, protein, or fluid. ${ }^{5}$ Unlike the modified Atkins diet, however, the low glycemic index treatment limits specific carbohydrates to those with glycemic indices of less than 50 (and those at $40-60 \mathrm{~g} /$ day).

Do these diets reduce seizure frequency? There is an abundance of data from both animals and humans that dietary manipulations of metabolism can positively affect seizure activity, both in the short and long term. In addition, there is evidence that diets can modify disease progression and may have neuroprotective properties. Certainly, some of the metabolic substrates elaborated by these diets have been shown in the laboratory to exert profound neuroprotective effects. Here we will discuss the evidence base for short- and long-term benefits of dietary therapies for epilepsy.

\section{SHORT-TERM BENEFITS}

\section{Animal evidence}

Ever since its clinical introduction in the 1920 s, the $\mathrm{KD}$ has fascinated investigators in terms of the underly- 
ing mechanisms of action. The earliest animal studies involving the $\mathrm{KD}$ focused on the acute effects of ketone bodies. In the 1930s, Keith ${ }^{6}$ was the first to demonstrate that, in rabbits, acetoacetate (ACA) was protective against seizures induced by thujone, a convulsant constituent found in many essential oils and an antagonist of $\mathrm{GABA}_{\mathrm{A}}$ receptors. $^{7}$ This intriguing observation was confirmed in the present century by Likhodii et al., ${ }^{8}$ who found that both ACA and acetone blocked seizure activity induced by maximal electroshock and pentylenetetrazole in rats. Acetone itself was also effective against chemically evoked atypical absence seizures. These investigators also demonstrated that acetone, when injected intraperitoneally, yielded plasma and CSF concentrations consistent with doses used to suppress seizures, supporting the unusual possibility that this volatile agent might mediate the clinical effects of the KD. Further evidence of the acute anticonvulsant effects of ACA was provided in an audiogenic seizure-susceptible mouse model that has been used in screening for investigational compounds for the treatment of epilepsy. ${ }^{9}$

Curiously, however, although such experiments have demonstrated the acute anticonvulsant properties of $\mathrm{ACA}$ and acetone in vivo, there are no studies indicating that the major ketone body, $\beta$-hydroxybutyrate (BHB), can exert similar effects. Furthermore, in vitro experiments appear to support the notion that, unlike the medications used in clinical practice to treat epilepsy patients, BHB is not an anticonvulsant compound. Thio et al. ${ }^{10}$ found that acute application of BHB did not affect standard measures of synaptic transmission in rat hippocampus in vitro. Over a wide concentration range (300 $\mu \mathrm{mol} / \mathrm{L}$ to $10 \mathrm{mmol} / \mathrm{L}$ ), BHB did not affect $\mathrm{GABA}_{\mathrm{A}}$ receptors, ionotropic glutamate receptors, or voltagegated sodium channels. Surprisingly, despite the well known in vivo effects, ACA likewise did not affect synaptic transmission in their study. These negative findings may reflect in part the fact that 1) ketone bodies were infused acutely, not chronically; 2) experiments were conducted in normal, not epileptic, brain; and 3) both culture and perfusion media contained glucose, which might be expected to counter a ketotic environment. Taken together, the experimental evidence for whether ketone bodies exert direct anticonvulsant actions remains unclear.

Much of the existing animal literature pertaining to the $\mathrm{KD}$ involves rodent studies in which various high-fat treatments are implemented prior to acute provocation with either electrical stimulation or chemoconvulsant administration. Most animal diets have modeled the classic long-chain triglyceride diet originally formulated by Wilder, ${ }^{2}$ and conform closely to either a $4: 1$ or approximately 6:1 ketogenic ratio of fats to carbohydrates plus protein (by weight). In general, irrespective of precise dietary formulation-as long as ketosis is seen, reflecting a shift from primarily glycolysis to intermediary metabolism-anticonvulsant effects have been observed. Thus, whether seizures are provoked by corneal electroshock, hydration electroshock, maximal electroshock, pentylenetetrazol, bicuculline, semicarbazide, kainate, flurothyl, or $6 \mathrm{~Hz}$ stimulation, chronic pretreatment with a KD appears to render anticonvulsant effects. ${ }^{11-22}$ However, historical observations in mouse models reflecting anticonvulsant efficacy of $4: 1$ or $6: 1$ KDs may be confounded by lack of control for intake of vitamins, minerals, and antioxidants. When a balanced KD was applied in acute seizure models, anticonvulsant efficacy was not actually seen in mouse pentylenetetrazol, kainate, or flurothyl models. ${ }^{23}$

With all of these studies, a number of experimental problems have precluded a greater understanding of the $\mathrm{KD}$ at a mechanistic level. Most of the problems relate to variable methodologies (e.g., use of calorie restriction, age at initiation, and duration of therapy, dietary ratios and formulations, timing of treatment, mode of seizure induction, etc.), such that cross-comparisons have been impossible to make. ${ }^{24,25}$ Furthermore, even in the face of prominent ketosis (i.e., $>4 \mathrm{mmol} / \mathrm{L}$ ), protection against acutely induced seizure activity in animal models is not universally seen, which once again emphasizes the controversy surrounding the effects of ketone bodies.

Thavendiranathan et $\mathrm{al}^{26}$ examined the effects of a medium-chain triglyceride diet against electroshock- and pentylenetetrazol-induced seizures, despite the fact that ketonemia was equivalent to or substantially higher than that observed with administration of a classic long-chain triglyceride diet. Moreover, they found that the mediumchain triglyceride diet was proconvulsant in the maximal electroshock and maximal pentylenetetrazol models. ${ }^{26}$

These studies, in attempting to mirror the clinical experience, suggest that dietary effects in controlling excitability in the brain extend beyond species boundaries, but they do little to enhance our knowledge of underlying mechanisms of action. Rather, they raise more issues and highlight the necessity of developing and studying a more clinically relevant model. One critical limitation is that these laboratory investigations have been conducted in normal, not epileptic, rodent brain. What is required is a chronic model of the KD in an animal with early-onset, medically refractory epilepsy and with a responsivity to a particular formulation of a high-fat diet that best recapitulates all of the essential elements of the human experience (Table 1). One could reasonably argue that a direct translation of clinical protocols to the rodent may not yield similar features, but this would be at least represent a good beginning.

\section{Human evidence}

Rapidity of response. For years, anecdotal evidence has described an occasional and remarkable re- 
Table 1. Animal Models of the Ketogenic Diet: Observations and Clinical Correlates

Observation in Animal Models

Seizure type

Age range

Calorie restriction

Diet type

Ketosis

Fat

Latency to KD effectiveness

Reversal of protective effect when KD discontinued
$\mathrm{KD}$ is effective in models employing a wide variety of seizure paradigms

Younger animals respond better to $\mathrm{KD}$

Increases seizure threshold

Classic and MCT diets both increase seizure threshold

A threshold level of ketosis is necessary but not sufficient to explain antiseizure effect

Better effectiveness with higher ketogenic ratios; uncertain if type of fat is a critical variable

Several days

Rapid (hours)
Clinical Correlate

$\mathrm{KD}$ is effective in many seizure types and epilepsy syndromes

Children extract and utilize ketones from blood more efficiently than older individuals

Associated with seizure reduction

In patients, classic and MCT KDs are equally efficacious

Ketosis is necessary but not sufficient

Practical concerns limit the ketogenic ratio; possible role of fat chain length and degree of saturation (e.g., PUFA)

Seizures may be seen during the pre$\mathrm{KD}$ fast or after a latency of days to weeks

Rapid (hours)

Reprinted with permission from Stafstrom, 1999. ${ }^{25}$

$\mathrm{KD}=$ ketogenic diet; $\mathrm{MCT}=$ medium chain triglycerides; PUFA $=$ polyunsaturated fatty acids .

sponse to the KD within hours of its initiation. It has been reported that fasting, even before actual ketogenic food is provided, can lead to a rapid improvement in seizures. ${ }^{27}$ More recent evidence has confirmed that the $\mathrm{KD}$ works quickly as an anticonvulsant. In a two-center study of 99 children who responded to the KD, the median time at which parents reported significant seizure reduction was after 5 days (range, $1-65$ days). ${ }^{28}$ Children who were fasted and improved did so more quickly, a median of 9 days more rapid $(p<0.01) .{ }^{28}$ This rapidity of response was also seen in a double-blind, placebocontrolled, crossover design study of the KD. ${ }^{29}$ In 20 children, the KD was provided with two fasting periods over just a 12-day period, resulting in a median reduction of 34 seizures per day, with $65 \%$ having a $>50 \%$ improvement. $^{29}$

Similarly, the modified Atkins diet also appears to work very quickly. In both children and adults, improvement is seen in most patients within 14 days. ${ }^{4}$ The data for the low glycemic index treatment in 20 children also indicate that an acute effect is present. ${ }^{30}$ The reason why this rapid response occurs is not clear and may indicate that fasting is a distinct mechanism of action of dietary therapies.

3- to 6-month data. One of the largest prospective studies to date evaluated the outcomes of 150 consecutive children started on the KD at Johns Hopkins Hospital from 1996 to $1998 .{ }^{31}$ At 6 months, $71 \%$ remained on the diet. Fifty-one percent had a $>50 \%$ reduction in seizures, and $32 \%$ had a $>90 \%$ reduction. No specific seizure type was preferentially improved, but the KD was somewhat less effective in children older than 8 years of age.

The strong evidence for short-term efficacy in multiple retrospective and prospective studies was recently summarized in a meta-analysis by Henderson et al. ${ }^{1}$ in 2006. In this review, $50 \%$ of children had at least a $50 \%$ reduction in seizures by 6 months with 20\% having a $>90 \%$ reduction. Although none of the studies in the meta-analysis had a control group, the evidence was interpreted as compelling for the benefits of the KD.

Since 2006, there have been several large studies that have further demonstrated the short-term effectiveness of the $\mathrm{KD}$, including some with control groups. ${ }^{29,32}$ In a 2008 study from London, ${ }^{32}$ children were randomly assigned to receive the KD after either 4 weeks (treatment group) or 16 weeks (control). Of the children receiving the diet, $38 \%$ had a $>50 \%$ reduction in seizures, compared to $6 \%$ of the control subjects $(p<0.0001){ }^{32}$ Perhaps even more dramatic, there was a $38 \%$ decrease in the mean number of seizures with the diet, compared with an increase of $37 \%$ in the control group. A doubleblinded study of children with Lennox-Gastaut syndrome compared a treatment (saccharin solution) to a placebo (glucose solution) arm during the first 12 days of the diet after fasting periods. ${ }^{29}$ Clinical seizures were reduced by 1.5 seizures per day with the saccharin solution, but this finding did not reach statistical significance $(p=0.07) .^{29}$

When analyzing all large studies (those with more than 20 subjects) just over the past decade, the results are quite similar to those reviewed by Henderson in 2006 
Table 2. Published Efficacy Studies of the Ketogenic Diet, Retrospective and Prospective, with 20 or More Patients, 1998-2008

\begin{tabular}{|c|c|c|c|c|c|}
\hline \multirow[b]{2}{*}{ Reference } & \multirow[b]{2}{*}{ Study Type } & \multirow[b]{2}{*}{ Patients, no. } & \multirow[b]{2}{*}{ Age Range, yr } & \multicolumn{2}{|c|}{ Seizure Improvement at $6 \mathrm{mo}, \%$} \\
\hline & & & & $>50 \%$ Reduction & $>90 \%$ Reduction \\
\hline Vining et al., ${ }^{75} 1998$ & Prosp. & 51 & $1-9$ & 53 & 29 \\
\hline Freeman et al., 1998 & Prosp. & 150 & $1-16$ & 51 & 32 \\
\hline Hassan et al., ${ }^{76} 1999$ & Retrosp. & 52 & $2-9$ & 67 & \\
\hline Kankirawatana et al., ${ }^{77} 2001$ & Prosp. & 35 & $0.2-13$ & & 75 \\
\hline Nordli et al., ${ }^{78} 2001$ & Retrosp. & 32 & $0.5-1.5$ & 55 & \\
\hline Kossoff et al., ${ }^{79} 2002$ & Retrosp. & 23 & $0.5-2$ & 72 & 39 \\
\hline Coppola et al., ${ }^{80} 2002$ & Prosp. & 56 & $1-23$ & 27 & \\
\hline Francois et al., ${ }^{81} 2003$ & Retrosp. & 29 & $0.3-12.5$ & 41 & \\
\hline Mady et al., ${ }^{82} 2003$ & Retrosp. & 45 & $12-19$ & 50 & 29 \\
\hline Klepper et al., ${ }^{83} 2004$ & Retrosp. & 111 & $0.1-18$ & 31 & 17 \\
\hline Vaisleib et al., ${ }^{84} 2004$ & Retrosp. & 54 & $2-14$ & 65 & \\
\hline Kang et al., ${ }^{85} 2005$ & Retrosp. & 199 & $0.5-17.5$ & 58 & \\
\hline Bergqvist et al., ${ }^{86} 2005$ & Prosp. & 48 & $1-14$ & $63 *$ & $38 *$ \\
\hline Eun et al., ${ }^{87} 2006$ & Retrosp. & 43 & $0.5-4$ & 81 & 63 \\
\hline Kossoff et al., ${ }^{88} 2007$ & Retrosp. & 30 & 4-24 & 63 & 23 \\
\hline Seo et al., ${ }^{89} 2007$ & Prosp. & 76 & $0.3-16$ & 79 & 49 \\
\hline Hamdy et al., ${ }^{90} 2007$ & Retrosp. & 90 & $0.3-14.8$ & 74 & 44 \\
\hline Kossoff et al., ${ }^{28} 2008$ & Retrosp. & 118 & $0.3-15$ & 71 & 43 \\
\hline Neal et al., ${ }^{32} 2008$ & Prosp. & 73 & $2-16$ & $38 *$ & $7 *$ \\
\hline Freeman et al., ${ }^{29} 2009$ & Prosp. & 20 & $1-10$ & 80 & \\
\hline Total & & 1,335 & $0.3-24$ & 56 & 24 \\
\hline
\end{tabular}

Prosp. $=$ prospective; Retrosp. $=$ retrospective.

*At 3 months.

(Table 2). After 6 months, $56 \%$ of 1335 patients had a $>50 \%$ seizure reduction and $24 \%$ had a $>90 \%$ seizure improvement.

Results from alternative diets. The modified Atkins diet and low glycemic index treatment have only recently come into clinical use, and definitive evidence of clinical effectiveness is not yet available. All studies of these diets to date have been limited to 6 months and report only short-term efficacy results. Nonetheless, the results are promising, and quite similar to the traditional KD.

The first prospective study of the modified Atkins diet in children aged 3 years to 16 years was reported in $2006 .{ }^{33}$ Of the 20 children, $13(65 \%)$ had a $>50 \%$ seizure reduction, $7(35 \%)$ had a $>90 \%$ improvement, and $4(20 \%)$ became seizure-free. In addition, 9/20 children were successfully able to reduce anticonvulsant medications. ${ }^{33}$ Now, after 6 years of continuous use documented in 100 children and adults with intractable epilepsy, the numbers remain surprisingly similar: $45 / 100$ have had at least a $50 \%$ reduction in their seizure frequency and $28 / 100$ have $>90 \%$ reduction. ${ }^{4}$

A study from Massachusetts General Hospital in 2005 described an even less restrictive diet, the low glycemic index treatment. ${ }^{30}$ In this study, 10 of 20 children had a $>90 \%$ seizure reduction despite an absence of urinary ketones and only low levels of serum ketones. A recent review from the same center reported on 60 patients, with a $38 \%$ responder rate after 1 month. ${ }^{5}$

Diet indications. Glucose transporter 1 (GLUT-1) deficiency syndrome and pyruvate dehydrogenase deficiency (PDHD) are now clearly indications for use of the $\mathrm{KD}$, which can be life-saving. Nonetheless, given that the majority of uses of the KD have been for epilepsy, understanding which epilepsy syndromes are more likely to respond than others remains a very important question. The past decade has witnessed a strong interest in finding these ideal epileptic disorders in which the KD may show higher seizure reduction rates than the available 3to 6-month efficacy data. These indications, which have been recently summarized in an international consensus statement on the $\mathrm{KD},{ }^{34}$ include infantile spasms, tuberous sclerosis complex, myoclonic-astatic epilepsy, severe myoclonic epilepsy of infancy (Dravet syndrome), and Rett syndrome. In addition, children in certain clinical situations appear to do remarkably well: those with gastrostomy tubes, infants formula-fed only, and those with concurrent vagus nerve stimulator therapy. ${ }^{34}$

\section{LONG-TERM BENEFITS}

\section{Animal evidence}

Neuroprotection and antiepileptogenesis. In approximately $20 \%$ of patients, anticonvulsant medications 
can be successfully discontinued without recrudescence of seizures. It is this observation that forms the basis for the intriguing hypothesis that a KD may possess neuroprotective or antiepileptogenic properties. Although several anecdotal accounts of an enduring effect of the KD exist, recent clinical studies more strongly suggest a potential antiepileptogenic effect of the KD. ${ }^{35,36}$ However, it is difficult to determine whether patients who remain seizure-free after cessation of a KD have had spontaneous remission of their epilepsy or have experienced a true direct antiepileptogenic (i.e., disease-modifying) effect. Moreover, it is not possible to account for the effects of blocking seizure activity over a prolonged duration.

To date, other than anecdotal reports, there are no data from controlled studies to indicate whether the KD is in fact neuroprotective or antiepileptogenic. In considering such specific effects, it should be noted that epilepsy can develop without overt evidence of neuronal injury, ${ }^{37}$ and neuronal damage does not necessarily lead to epilepsy. ${ }^{38}$ Hence, the terms neuroprotection and antiepileptogenesis are not necessarily synonymous.

Despite the lack of clinical data, there are a growing number of animal and in vitro studies supporting both neuroprotective and antiepileptogenic effects of the KD. Hori et al. ${ }^{39}$ were the first to demonstrate the long-term effects of a KD in a chronic epilepsy model. In this study, adult male rats were first subjected to electrical kindling and then, 10 days after being fully kindled, were treated with either a 4:1 KD or standard rodent chow. For 5 weeks, after-discharge threshold and duration and stage 5 seizure threshold and duration were assessed on a weekly basis. Additionally, during the 3rd week, the investigators studied the influence of a KD on learning and memory, using the standard Morris water-maze and open-field tests. The KD-fed animals exhibited an elevated afterdischarge threshold and stage 5 seizure threshold relative to controls, but this effect was transient, lasting only 2 weeks. Notably, this loss of protection occurred despite the maintenance of ketonemia. Furthermore, KD treatment did not adversely affect performance on either the water-maze or the open-field test.

This last observation contrasts with a more recent study indicating that, after lithium-pilocarpine-induced status epilepticus, KD treatment significantly impaired visuospatial learning and memory, compared with rats that were fed a regular diet. ${ }^{40}$ Moreover, animals fed a KD exhibited significant impairment in brain growth. It remains unclear whether this was due to use of a much higher ketogenic ratio (8.6:1) than is standard practice, the long-term consequences of impaired food intake, or both. ${ }^{41}$ Taken together, the evidence does not yet establish whether the KD preserves, enhances, or disturbs cognitive functioning in rodent models.

The first evidence that a KD can retard epileptogenesis in an animal model was demonstrated by MullerSchwarze et al. ${ }^{20}$ In this study, rats were first subjected to kainate-induced status epilepticus, and then were treated with a KD. After the latent period, spontaneous recurrent seizures were recorded, and routine hippocampal histology and Timm histochemistry (to label zinc-containing mossy fiber terminals in the dentate gyrus) were conducted on animals after an 8-week treatment period. Seizure frequency and duration were both significantly lower in the KD-treated group than in the control group. Furthermore, although the KD did not appear to alter the degree of neuronal damage in either the CA1 or the CA3 subfields of the hippocampus, there was a substantial reduction in the extent of Timm labeling (indicative of mossy fiber sprouting).

In contrast, preliminary reports addressing the effects of a KD in the rat kainic acid model yielded somewhat contradictory results. Bough et al. ${ }^{42}$ treated adult Sprague-Dawley rats with a KD prior to treatment with kainic acid (administered either subcutaneously or intraperitoneally). Rats fed a KD experienced more severe seizures and greater mortality than did the control animals, and this exacerbation of kainic acid-induced seizures was seen irrespective of route of administration. Similarly, in immature (P20) Sprague-Dawley rats, Ko et al. $^{43}$ found that a 2-week period of KD pretreatment exacerbated hippocampal neuronal damage in the CA1 and CA3 subfields 72 hours after kainic acid injection; no significant differences in seizure latencies were observed between the two groups.

The seemingly conflicting results of these studies may be explained in part by differences in timing of $\mathrm{KD}$ initiation. In the study of Muller-Schwarze et al., ${ }^{20}$ the $\mathrm{KD}$ was initiated after spontaneous recurrent seizures manifested, whereas in the study by Bough et al., ${ }^{42}$ the $\mathrm{KD}$ was administered prior to induction of acute seizures with kainic acid. In the case of KD treatment prior to kainic acid exposure in immature rats, ${ }^{43}$ there may be developmental differences as well.

The conflicting results of KD treatment in rat models of status epilepticus have not been observed in studies involving mice. Noh et al. ${ }^{21}$ examined the effects of a $\mathrm{KD}$ on kainate-induced hippocampal cell death in mice. Males of an outbred strain of Swiss mice (Institute for Cancer Research or ICR mice, otherwise known as Caesarean Derived-1 or CD-1) were administered kainate (25 mg/kg, intraperitoneally) after a 4-week treatment with a $4: 1 \mathrm{KD}$ formulation. Histological assessment of injury, using routine cresyl violet staining, in situ DNA nick-end (i.e., TUNEL) labeling, and proapoptotic caspase-3 immunoreactivity, was done at 2 days following kainate treatment. The KD-fed mice exhibited significantly prolonged seizure latency to kainate, but no changes in behavioral seizure severity were reported as compared to control animals. The kainate-treated group 
demonstrated typical cellular loss and pyknosis in the CA1 and CA3 hippocampal subfields, and there was marked TUNEL positivity in these regions. In contrast, KD treatment prevented cell loss and increase in DNA nick-end labeling; notably, there was a striking decrease in caspase 3 immunoreactivity in KD-fed animals. Taken together, these results indicate that pretreatment with a $\mathrm{KD}$ protected against kainate-induced neuronal death in mice.

In contrast to induced models, what are the effects of a KD in genetically determined epilepsy models? The seizure-susceptible EL mouse is an inbred strain that has been extensively described as a model of multifactorial idiopathic partial epilepsy with secondary generalization. ${ }^{44}$ Generalized seizures generally manifest by the second postnatal month and persist throughout later life, but environmental stimulation such as repetitive handling can induce seizures and facilitate epileptogenesis beginning at $\mathrm{P} 30 .^{45}$

Todorova et al. ${ }^{46}$ treated male EL mice with a $4.75: 1$ ketogenic formula; over a 10 -week period, mice were tested weekly for seizure activity induced by handling. Seizure susceptibility scores in the KD group were significantly lower than in the control mice after 3 weeks, but this difference disappeared by week 7. Overall, KD treatment delayed seizure onset in young EL mice by 1 month, and (similar to what had been reported by Hori et al. ${ }^{39}$ in the kindling model) seizure protection was transient.

More recently, Fenoglio et al. ${ }^{47}$ reported that a $6.3: 1$ KD reduced seizure frequency in Kcnal-null mice lacking the gene encoding the delayed rectifier potassium channel $\alpha$ subunit, Kv1.1, which is important in regulating neuronal membrane excitability. This observation may be of particular interest, given that 1) Kcnal-null mice exhibit similar progressive histological changes in the hippocampus to those observed both in human epileptic tissue and in many animal models ${ }^{48}$ of temporal lobe epilepsy and 2) the Kcnal gene is one of only two epilepsy genes in a developmental animal model that has a homolog in a human epileptic condition. ${ }^{49}$

Potential Neuroprotective Mechanisms. Ketone bodies and polyunsaturated fatty acids (PUFAs) (metabolic substrates that are both elevated in epileptic patients treated with the $\mathrm{KD}^{50,51}$ ) have been shown to exert neuroprotective activity in neurodegenerative conditions associated with impaired mitochondrial function. ${ }^{52-57}$ To date, in acute seizure or chronic epilepsy models, it is unknown whether PUFAs can exert protective effects. Willis et al. ${ }^{58}$ administered the PUFAs eicosapentanoic acid and docosahexaenoic acid adjunctively with standard chow to normal mice, and evaluated the effects of such PUFA supplementation against acute provocation flurothyl, pentylenetetrazol, $6 \mathrm{~Hz}$, and kainate models. Neither docosahexaenoic acid nor eicosapentanoic acid feeding demonstrated anticonvulsant or neuroprotective activity, despite significant alterations in the fatty acid profile in both plasma and brain. What remains unclear is whether chronic administration of PUFAs to epileptic animals would produce an altogether different effect.

Some of the salient mechanisms of neuroprotection likely involve a reduction in mitochondrial free radical production, which would decrease oxidative stress, and potentially neuronal injury. ${ }^{57,59-61}$ Although it remains unclear how the KD specifically produces an anticonvulsant action, the neuroprotective properties of both the $\mathrm{KD}$ and of ketone bodies suggest that enhanced ATP synthesis, ${ }^{62,63}$ increased mitochondrial uncoupling, ${ }^{64}$ suppression of reactive oxygen species production ${ }^{59,60}$, and elevated glutathione levels ${ }^{61}$ may prevent epileptogenesis and likely as a consequence prevent recurrent seizure activity. ${ }^{65}$

Another hypothesis for the anticonvulsant action of the KD posits that increased ATP synthesis should produce a positive bioenergetic balance, allowing stabilization of the resting membrane potential via enhanced activity of $\mathrm{Na}+, \mathrm{K}+$, and ATPase. ${ }^{66}$ Several decades ago, DeVivo et al. ${ }^{63}$ reported that the KD increased the total quantity of bioenergetic substrates (such as ATP and phosphocreatine) and elevated the energy charge in rat brain. These changes were thought to stabilize the cell membrane, especially in the face of excessive excitation and energy demand. Consistent with these observations, a subsequent human study using magnetic resonance spectroscopic techniques indicated that patients with epilepsy fed a KD had elevated levels of both phosphocreatine and creatine in the brain. ${ }^{67}$

Recent animal studies have further supported the bioenergetic theory of KD action. Using cDNA microarray technology, increased expression of the mitochondrial ATP synthase $\beta$, subunit $\mathrm{D}$, was reported after KD treatment in mice. ${ }^{68}$ In the most comprehensive study of this kind to date, the KD was found to enhance mitochondrial biogenesis and significantly increase the number of transcripts encoding energy metabolism genes in rats. ${ }^{62}$ This increase in bioenergetic capacity enabled hippocampal slices from these animals to better withstand metabolic challenge from low glucose exposure. Taken together, the prevailing notion is that increased energy production and reserve capacity enable greater resistance to neuronal hyperexcitability and injury.

Other mechanistic considerations. Glucose restriction has long been implicated in the mechanisms of KD action, ${ }^{57,66,69}$ based on the clinical observation of mild glucopenia in treated epilepsy patients. Thus, it is of considerable interest that Garriga-Canut et al. ${ }^{70} \mathrm{dem}$ onstrated that 2-deoxyglucose (2-DG) (an inhibitor of phosphoglucose isomerase) potently inhibited seizure progression in the rat kindling model of temporal lobe epilepsy, and decreased the expression of brain-derived 
neurotrophic factor BDNF and its principal receptor, TrkB. These effects were linked to the induction of the transcription factor NRSF (neuron restrictive silencing factor), a master negative regulator of neuronal genes. In preclinical screening models, Stafstrom et al. ${ }^{71}$ evaluated the effects of 2-DG in multiple in vitro and in vivo models and found that 2-DG exerted acute anticonvulsant and chronic antiepileptic actions with a novel pattern of effectiveness.

In a related direction, Lian et al. ${ }^{72}$ demonstrated that fructose-1,6-bisphosphate, a metabolite that shifts the metabolism of glucose from glycolysis to the pentose phosphate pathway, exhibits potent anticonvulsant activity in several rat models of acute seizures (i.e., pilocarpine, kainic acid, and pentylenetetrazole); efficacy in these models exceeded that of 2-DG and KD treatment.

Collectively, emerging data indicate that the overall strategy of limiting glycolytic flux may be a powerful way of preventing acute seizures and perhaps epileptogenesis as well. It remains to be established whether glucose restriction allows for a compensatory increase in fatty acid oxidation leading to elevated ketone body production, or to enhanced flux of tricarboxylic acid intermediates (e.g., by administering anaplerotic substrates that can replenish a depleted tricarboxylic acid pool; K. Borges, personal communication). Taken together, the evidence to date strongly suggests that metabolic regulation is a potential therapeutic strategy for seizure suppression and modulation of epileptogenesis.

\section{Human evidence}

KD for 3 to 6 years. A common misconception is that the KD not only must be discontinued after 2 years due to side effects, but also that efficacy will wane over time. This does not appear to be true, based on recent studies that examined the long-term efficacy of the KD after periods longer than 6 to 12 months. In the largest long-term study to date, children from the original 150 patient cohort first reported in $1998^{31}$ were contacted again after 3 to 6 years. ${ }^{35}$ Using an intent-to-treat analysis, $65(43 \%)$ had a $>50 \%$ seizure reduction when followed, including many who were now off the KD. ${ }^{35}$ For those who remained on the diet for more than 4 years, 13 of $15(87 \%)$ had $>50 \%$ reduction in seizures.

These benefits were noted not only in those who remained on the KD for extended periods of time. Of these original 150 children, 28 discontinued the KD before 6 months of use (most because of KD inefficacy) and were then analyzed separately. ${ }^{36}$ Although one would expect them to be doing poorly 3 to 6 years later, in fact 12 of the 28 children $(43 \%)$ were $>50 \%$ improved, which was similar to the overall population. ${ }^{36}$ Five of these children had surgery to improve their epilepsy, but (surprisingly) seven did not.
KD for 6 to 12 years. The KD appears to be effective even beyond 6 years. At Johns Hopkins Hospital, of approximately 600 children who have been started on the diet since 1994, 28 children had been continuously on the KD for more than 6 years (range, 6-12 years) when analyzed in $2006 .{ }^{73}$ The majority of these children remained on the KD because of dramatic seizure reduction and then, because this did not reach the level of seizure freedom, the KD was maintained. Even after these extended periods, all children maintained large urinary ketosis and control was stable, with only periodic breakthrough seizures. Of these 28 children, 24 (86\%) were reported as having $>90 \%$ seizure reduction. ${ }^{73}$

Beyond two decades. Although anecdotal cases of continuous KD use for decades are known from children treated prior to 1965 , no such cases have been reported in the medical literature. This changed in 2008 with the case report of a 29-year-old man with tuberous sclerosis complex who had started the KD at age 6 years of age. ${ }^{74}$ This patient's family had continuously provided the KD for nearly a decade without neurologist or dietitian supervision. To date, he remains on a 3:1 KD with large ketosis and $>90 \%$ seizure reduction, with approximately 1-2 seizures per year. His parents provide his KD, which he brings daily to work, and he does not appear to have tolerability issues.

Results from this study and the others discussed in this review suggest that the benefits of the KD can be seen long term and do not diminish over time. They may even persist for years after the KD is stopped. For patients in whom the KD proves useful and necessary long-term, it may be reasonable (given the potential side effects of the $\mathrm{KD}$ from extended use, including kidney stones, bone fractures, and decreased linear growth ${ }^{73}$ ) to consider changing to the modified Atkins treatment or low glycemic index treatment after several years. For now, however, because these alternative diets have only recently been introduced, we do not yet know the long-term efficacy profile, nor whether they truly have fewer longterm side effects than the $\mathrm{KD}$.

\section{CONCLUSION}

In summary, the traditional and alternative KDs appear to show significant immediate and short-term benefits. This effect is seen in both animal and human studies. Because it has been in use for nearly a century, the $\mathrm{KD}$ has also had sufficient time to demonstrate long-term clinical benefits in those who maintain it and - notablyeven in those who discontinue it. Recent animal studies have also confirmed this finding that the KD and its metabolic substrates may exert neuroprotective properties. Further studies of dietary therapies are necessary and ongoing. 


\section{REFERENCES}

1. Henderson CB, Filloux FM, Alder SC, Lyon JL, Caplin DA. Efficacy of the ketogenic diet as a treatment option for epilepsy: meta-analysis. J Child Neurol 2006;21:193-198.

2. Wilder RM. The effects of ketonemia on the course of epilepsy. Mayo Clin Bull 1921;2:307-308.

3. Freeman JM, Kossoff EH, Hartman AL. The ketogenic diet: one decade later. Pediatrics 2007;119:535-543.

4. Kossoff EH, Dorward JL. The modified Atkins diet. Epilepsia 2008;49 Suppl 8:37-41.

5. Pfeifer HH, Lyczkowski DA, Thiele EA. Low glycemic index treatment: implementation and new insights into efficacy. Epilepsia 2008:49 Suppl 8:42-45.

6. Keith HM. Factors influencing experimentally produced convulsions. Arch Neurol Psychiatry 1933;29:148-154.

7. Höld KM, Sirisoma NS, Ikeda T, Narahashi T, Casida JE. $\alpha$-Thujone (the active component of absinthe): $\gamma$-aminobutyric acid type A receptor modulation and metabolic detoxification. Proc Natl Acad Sci U S A 2000;97:3826-3831.

8. Likhodii SS, Serbanescu I, Cortez MA, Murphy P, Snead OC 3rd, Burnham WM. Anticonvulsant properties of acetone, a brain ketone elevated by the ketogenic diet. Ann Neurol 2003;54:219-226.

9. Rho JM, White HS, Anderson G, Donevan S. Acetoacetate, acetone, and dibenzylamine (a contaminant in L- $(+)-\beta$-hydroxybutyrate) exhibit direct anticonvulsant actions in vivo. Epilepsia 2002;43:358-361.

10. Thio LL, Wong M, Yamada KA. Ketone bodies do not directly alter excitatory or inhibitory hippocampal synaptic transmission. Neurology 2000;54:325-331.

11. Millichap JG, Jones JD, Rudis BP. Mechanisms of anticonvulsant action of ketogenic diet. Am J Dis Child 1964;107:593-604.

12. Uhlemann ER, Neims AH. Anticonvulsant properties of the ketogenic diet in mice. J Pharmacol Exp Ther 1972;180:231-238.

13. Appleton DB, DeVivo DC. An animal model of the ketogenic diet. Epilepsia 1974;15:211-227.

14. Mahoney AW, Hendricks DG, Bernhard N, Sisson DV. Fasting and ketogenic diet effects on audiogenic seizures susceptibility of magnesium deficient rats. Pharmacol Biochem Behav 1983;18: 683-687.

15. Nakazawa M, Kodama S, Matsuo T. Effects of ketogenic diet on electroconvulsive threshold and brain contents of adenosine nucleotides. Brain Dev 1983;5:375-380.

16. Otani K, Yamatodani A, Wada H, Mimaki T, Yabuuchi H. Effect of ketogenic diet on convulsive threshold and brain monoamine levels in young mice [In Japanese]. No To Hattasu 1984;16:196204.

17. Bough KJ, Eagles DA. A ketogenic diet increases the resistance to pentylenetetrazole-induced seizures in the rat. Epilepsia 1999;40: $138-143$.

18. Bough KJ, Matthews PJ, Eagles DA. A ketogenic diet has different effects upon seizures induced by maximal electroshock and by pentylenetetrazole infusion. Epilepsy Res 2000;38:105-114.

19. Rho JM, Kim DW, Robbins CA, Anderson GD, Schwartzkroin PA. Age-dependent differences in flurothyl seizure sensitivity in mice treated with a ketogenic diet. Epilepsy Res 1999;37:233-240.

20. Muller-Schwarze AB, Tandon P, Liu Z, Yang Y, Holmes GL, Stafstrom CE. Ketogenic diet reduces spontaneous seizures and mossy fiber sprouting in the kainic acid model. Neuroreport 1999; 10:1517-1522.

21. Noh HS, Kim YS, Lee HP, et al. The protective effect of a ketogenic diet on kainic acid-induced hippocampal cell death in the male ICR mice. Epilepsy Res 2003;53:119-128.

22. Hartman AL, Lyle M, Rogawski MA, Gasior M. Efficacy of the ketogenic diet in the 6-Hz seizure test. Epilepsia 2008;49:334339.

23. Samala R, Willis S, Borges K. Anticonvulsant profile of a balanced ketogenic diet in acute mouse seizure models. Epilepsy Res 2008; 81:119-127.

24. Stafstrom CE. Animal models of the ketogenic diet: what have we learned, what can we learn? Epilepsy Res 1999;37:241-259.

25. Stafstrom CE. Dietary approaches to epilepsy treatment: old and new options on the menu. Epilepsy Curr 2004;4:215-222.
26. Thavendiranathan P, Mendonca A, Dell C, et al. The MCT ketogenic diet: effects on animal seizure models. Exp Neurol 2000; 161:696-703.

27. Freeman JM, Vining EPG. Seizures decrease rapidly after fasting: preliminary studies of the ketogenic diet. Arch Pediatr Adolesc Med 1999;153:946-949.

28. Kossoff EH, Laux LC, Blackford R, et al. When do seizures improve with the ketogenic diet? Epilepsia 2008;49:329-333.

29. Freeman JM, Vining EPG, Kossoff EH, Pyzik PL, Ye X, Goodman SN. A blinded, crossover study of the ketogenic diet. Epilepsia 2009;50:322-325.

30. Pfeifer HH, Thiele EA. Low-glycemic-index treatment: a liberalized ketogenic diet for treatment of intractable epilepsy. Neurology 2005;65:1810-1812.

31. Freeman JM, Vining EPG, Pillas DJ, Pyzik PL, Casey JC, Kelly MT. The efficacy of the ketogenic diet-1998: a prospective evaluation of intervention in 150 children. Pediatrics 1998;102:13581363.

32. Neal EG, Chaffe H, Schwartz RH, et al. The ketogenic diet in the treatment of epilepsy in children: a randomised, controlled trial. Lancet Neurol 2008;7:500-506.

33. Kossoff EH, McGrogan JR, Bluml RM, Pillas DJ, Rubenstein JE, Vining EP. A modified Atkins diet is effective for the treatment of intractable pediatric epilepsy. Epilepsia 2006;47:421-424.

34. Kossoff EH, Zupec-Kania BA, Amark PE, et al. Optimal clinical management of children receiving the ketogenic diet: recommendations of the international ketogenic diet study group. Epilepsia 2008 Sept 23 [Epub ahead of print].

35. Hemingway C, Freeman JM, Pillas DJ, Pyzik PL. The ketogenic diet: a 3- to 6-year follow-up of 150 children enrolled prospectively. Pediatrics 2001;108:898-905.

36. Marsh EB, Freeman JM, Kossoff EH, et al. The outcome of children with intractable seizures: a 3- to 6-year follow-up of 67 children who remained on the ketogenic diet less than one year. Epilepsia 2006;47:425-430.

37. Raol YS, Budreck EC, Brooks-Kayal AR. Epilepsy after early-life seizures can be independent of hippocampal injury. Ann Neurol 2003;53:503-511.

38. Baram TZ, Eghbal-Ahmadi M, Bender RA. Is neuronal death required for seizure-induced epileptogenesis in the immature brain? Prog Brain Res 2002;135:365-375.

39. Hori A, Tandon P, Holmes GL, Stafstrom CE. Ketogenic diet: effects on expression of kindled seizures and behavior in adult rats. Epilepsia 1997;38:750-758.

40. Zhao Q, Stafstrom CE, Fu DD, Hu Y, Holmes GL. Detrimental effects of the ketogenic diet on cognitive function in rats. Pediatr Res 2004;55:498-506.

41. Cunnane SC, Likhodii SS. Claims to identify detrimental effects of the ketogenic diet on cognitive function in rats. Pediatr Res 2004; 56:663-664.

42. Bough KJ, Fetner JD, Eagles DA. A ketogenic diet exacerbates kainate-induced seizures in the rat. Soc Neurosci Abstr 1998;24: 1208.

43. Ko TS, Soo AC, Kim DW, Kim KJ. Ketogenic diet: effects on hippocampal c-fos expression and neuronal death after kainic acidinduced seizures in immature rats. Epilepsia 1999;40 Suppl 7:79 (abstract).

44. Brigande JV, Wieraszko A, Albert MD, Balkema GW, Seyfried TN. Biochemical correlates of epilepsy in the E1 mouse: analysis of glial fibrillary acidic protein and gangliosides. J Neurochem 1992;58:752-760.

45. Todorova MT, Burwell TJ, Seyfried TN. Environmental risk factors for multifactorial epilepsy in EL mice. Epilepsia 1999;40: $1697-1707$

46. Todorova MT, Tandon P, Madore RA, Stafstrom CE, Seyfried TN The ketogenic diet inhibits epileptogenesis in EL mice: a genetic model for idiopathic epilepsy. Epilepsia 2000;41:933-940.

47. Maganti R, Allen C, Wilke J, Milligan H, Rho JM, Fenoglio KA. Ketogenic diet treatment improves circadian rhythmicity and abolishes seizure periodicity in epileptic Kcnal-null mice. Epilepsia 2009 (in press).

48. Wenzel HJ, Vacher H, Clark E, et al. Structural consequences of 
Kcnal gene deletion and transfer in the mouse hippocampus. Epilepsia 2007;48:2023-2046.

49. Zuberi SM, Eunson LH, Spauschus A, et al. A novel mutation in the human voltage-gated potassium channel gene (Kv1.1) associates with episodic ataxia type 1 and sometimes with partial epilepsy. Brain 1999;122:817-825.

50. Freeman J, Veggiotti P, Lanzi G, Tagliabue A, Perucca E; Institute of Neurology IRCCS C. Mondino Foundation. The ketogenic diet: from molecular mechanisms to clinical effects. Epilepsy Res 2006; 68:145-180.

51. Fraser DD, Whiting S, Andrew RD, Macdonald EA, Musa-Veloso $\mathrm{K}$, Cunnane SC. Elevated polyunsaturated fatty acids in blood serum obtained from children on the ketogenic diet. Neurology 2003;60:1026-1029.

52. Kashiwaya Y, Takeshima T, Mori N, Nakashima K, Clarke K, Veech RL. D- $\beta$-hydroxybutyrate protects neurons in models of Alzheimer's and Parkinson's disease. Proc Natl Acad Sci U S A 2000;97:5440-5444.

53. García O, Massieu L. Strategies for neuroprotection against L-trans-2,4-pyrrolidine dicarboxylate-induced neuronal damage during energy impairment in vitro. J Neurosci Res 2001;64:418428.

54. Veech RL, Chance B, Kashiwaya Y, Lardy HA, Cahill GF Jr. Ketone bodies, potential therapeutic uses. IUBMB Life 2001;51: 241-247.

55. Noh HS, Hah YS, Nilufar R, et al. Acetoacetate protects neuronal cells from oxidative glutamate toxicity. J Neurosci Res 2006;83: 702-709.

56. Bazan NG. Omega-3 fatty acids, pro-inflammatory signaling and neuroprotection. Curr Opin Clin Nutr Metab Care 2007;10:136141.

57. Maalouf M, Rho JM, Mattson MP. The neuroprotective properties of calorie restriction, the ketogenic diet, and ketone bodies. Brain Res Rev 2008 Sep 25 [Epub ahead of print].

58. Willis S, Samala R, Rosenberger TA, Borges K. Eicosapentaenoic and docosahexaenoic acids are not anticonvulsant or neuroprotective in acute mouse seizure models. Epilepsia 2009;50:138-142.

59. Kim do Y, Davis LM, Sullivan PG, et al. Ketone bodies are protective against oxidative stress in neocortical neurons. J Neurochem 2007;101:1316-1326.

60. Maalouf M, Sullivan PG, Davis L, Kim DY, Rho JM. Ketones inhibit mitochondrial production of reactive oxygen species following glutamate excitotoxicity by increasing NADH oxidation. Neuroscience 2007;145:256-264.

61. Jarrett SG, Milder JB, Liang LP, Patel M. The ketogenic diet increases mitochondrial glutathione levels. J Neurochem 2008; 106:1044-1051.

62. Bough KJ, Wetherington J, Hassel B, et al. Mitochondrial biogenesis in the anticonvulsant mechanism of the ketogenic diet. Ann Neurol 2006;60:223-235.

63. DeVivo DC, Leckie MP, Ferrendelli JS, McDougal DB Jr. Chronic ketosis and cerebral metabolism. Ann Neurol 1978;3:331-337.

64. Sullivan PG, Rippy NA, Dorenbos K, Concepcion RC, Agarwal AK, Rho JM. The ketogenic diet increases mitochondrial uncoupling protein levels and activity. Ann Neurol 2004;55:576-580.

65. Acharya MM, Hattiangady B, Shetty AK. Progress in neuroprotective strategies for preventing epilepsy. Prog Neurobiol 2008;84: 363-404.

66. Bough KJ, Rho JM. Anticonvulsant mechanisms of the ketogenic diet. Epilepsia 2007;48:43-58.

67. Pan JW, Bebin EM, Chu WJ, Hetherington HP. Ketosis and epilepsy: 31P spectroscopic imaging at 4.1 T. Epilepsia 1999;40:703707.

68. Noh HS, Lee HP, Kim DW, et al. A cDNA microarray analysis of gene expression profiles in rat hippocampus following a ketogenic diet. Brain Res Mol Brain Res 2004;129:80-87.
69. Greene AE, Todorova MT, McGowan R, Seyfried TN. Caloric restriction inhibits seizure susceptibility in epileptic EL mice by reducing blood glucose. Epilepsia 2001;42:1371-1378.

70. Garriga-Canut M, Schoenike B, Qazi R, et al. 2-Deoxy-D-glucose reduces epilepsy progression by NRSF-CtBP-dependent metabolic regulation of chromatin structure. Nat Neurosci 2006;9:1382-1387.

71. Stafstrom CE, Ockuly JC, Murphree L, Valley MT, Roopra A, Sutula TP. Anticonvulsant and antiepileptic actions of 2-deoxy-Dglucose in epilepsy models. Ann Neurol 2009 (in press).

72. Lian XY, Khan FA, Stringer JL. Fructose-1,6-bisphosphate has anticonvulsant activity in models of acute seizures in adult rats. J Neurosci 2007;27:12007-12011.

73. Groesbeck DK, Bluml RM, Kossoff EH. Long-term use of the ketogenic diet in the treatment of epilepsy. Dev Med Child Neurol 2006;48:978-981.

74. Kossoff EH, Turner Z, Bergey GK. Home-guided use of the ketogenic diet in a patient for more than 20 years. Pediatr Neurol 2007;36:424-425.

75. Vining EP, Freeman JM, Ballaban-Gil K, et al. A multicenter study of the efficacy of the ketogenic diet. Arch Neurol 1998;55:14331437.

76. Hassan AM, Keene DL, Whiting SE, Jacob PJ, Champagne JR, Humphreys P. Ketogenic diet in the treatment of refractory epilepsy in childhood. Pediatr Neurol 1999;21:548-552.

77. Kankirawatana P, Jirapinyo P, Kankirawatana S, Wongarn R, Thamanasiri N. Ketogenic diet: an alternative treatment for refractory epilepsy in children. J Med Assoc Thai 2001;84:1027-1032.

78. Nordli DR Jr, Kuroda MM, Carroll J, et al. Experience with the ketogenic diet in infants. Pediatrics 2001;108:129-133.

79. Kossoff EH, Pyzik PL, McGrogan JR, Vining EP, Freeman JM Efficacy of the ketogenic diet for infantile spasms. Pediatrics 2002; 109:780-783.

80. Coppola G, Veggiotti P, Cusmai R, et al. The ketogenic diet in children, adolescents and young adults with refractory epilepsy: an Italian multicentric experience. Epilepsy Res 2002;48:221-227.

81. François LL, Manel V, Rousselle C, David M. Ketogenic regime as anti-epileptic treatment: its use in 29 epileptic children [In French]. Arch Pediatr 2003;10:300-306.

82. Mady MA, Kossoff EH, McGregor AL, Wheless JW, Pyzik PL, Freeman JM. The ketogenic diet: adolescents can do it, too. Epilepsia 2003;44:847-851.

83. Klepper J, Leiendecker B, Riemann E, Baumeister FA. The ketogenic diet in German-speaking countries: update 2003 [In German]. Klin Padiatr 2004;216:277-285.

84. Vaisleib II, Buchhalter JR, Zupanc ML. Ketogenic diet: outpatient initiation, without fluid, or caloric restrictions. Pediatr Neurol 2004;31:198-202.

85. Kang HC, Kim YJ, Kim DW, Kim HD. Efficacy and safety of the ketogenic diet for intractable childhood epilepsy: Korean multicentric experience. Epilepsia 2005;46:272-279.

86. Bergqvist AG, Schall JI, Gallagher PR, Cnaan A, Stallings VA. Fasting versus gradual initiation of the ketogenic diet: a prospective, randomized clinical trial of efficacy. Epilepsia 2005;46:18101819.

87. Eun SH, Kang HC, Kim DW, Kim HD. Ketogenic diet for treatment of infantile spasms. Brain Dev 2006;28:566-571.

88. Kossoff EH, Pyzik PL, Rubenstein JE, et al. Combined ketogenic diet and vagus nerve stimulation: rational polytherapy? Epilepsia 2007; 48:77-81.

89. Seo JH, Lee YM, Lee JS, Kang HC, Kim HD. Efficacy and tolerability of the ketogenic diet according to lipid:nonlipid ratios-comparison of 3:1 with 4:1 diet. Epilepsia 2007;48:801805.

90. Hamdy RF, Turner Z, Pyzik PL, Kossoff EH. Lack of influence of body mass index on the efficacy of the ketogenic diet. J Child Neurol 2007;22:1167-1171. 